\title{
Suppurative Arthritis
}

National Cancer Institute

\section{Source}

National Cancer Institute. Suppurative Arthritis. NCI Thesaurus. Code C118475.

Acute inflammation of one or more joints caused by the presence of pus within the joint cavity. 